05

\title{
Адгезия между жестким индентором и упругим полупространством для несжимаемых градиентных сред с высоким показателем градиентности
}

\author{
(C) Я.А. Ляшенко, ${ }^{1,2}$ В.Л. Попов ${ }^{1,3,4}$ \\ ${ }^{1}$ Берлинский технический университет, \\ 10623 Берлин, Германия \\ ${ }^{2}$ Сумский государственный университет, \\ 40007 Сумы, Украина \\ ${ }^{3}$ Национальный исследовательский Томский государственный университет, \\ 634050 Томск, Россия \\ ${ }^{4}$ Национальный исследовательский Томский политехнический университет, \\ 634050 Томск, Россия \\ e-mail: i.liashenko@tu-berlin.de
}

Поступило в Редакцию 7 апреля 2018 г.

В окончательной редакции 4 ноября 2019 г.

Принято к публикации 25 ноября 2019 г.

Рассмотрена контактная задача цилиндрического индентора и градиентной среды с учетом адгезионных сил между поверхностями контактирующих тел. Найдено аналитическое решение задачи для случая градиентной среды со степенной зависимостью упругого модуля от нормальной координаты. Подробно описан случай несжимаемых сред со значением коэффициента Пуассона 1/2. Показано, что в этом случае поведение системы может качественным образом отличаться от случая однородных сред. Рассмотрено влияние шероховатости поверхности, которая моделируется в виде аксиально-симметричной „волнистости“. Отдельно рассмотрен контакт параболического индентора и градиентной среды, показаны отличия от случая цилиндра.

Ключевые слова: адгезионный контакт, градиентные среды, метод редукции размерности, коэффициент Пуассона, индентирование.

DOI: 10.21883/JTF.2020.05.49176.140-18

\section{Введение}

Адгезия играет большую роль в технике, биологии и медицине [1], в связи с чем проводится активное изучение адгезионного контакта [2-6]. В частности, в последнее время большое значение уделяется изучению механических свойств градиентных сред различной природы. В таких средах параметры материала изменяются по его толщине. Если закон изменения параметров задается аналитически, то становится возможным решать стандартные задачи теории упругости, получая при этом практически важные нетривиальные результаты. В частности, градиентные среды, в которых модуль упругости увеличивает свое значение с глубиной (имеет малое значение на поверхности объекта), используются для создания защитных шлемов, защитных накладок на суставы, бронежилетов, и т.д. Такие защитные средства позволяют более плавно во времени поглощать кинетическую энергию объекта при механическом воздействии на них извне. Подобная ситуация будет наблюдаться и для случая, когда модуль упругости с глубиной уменьшается, только в этом случае начальная деформация приходится на внутреннюю поверхность защитного средства. В описанных применениях должна либо присутствовать вязкая диссипация энергии, либо защитные средства при превышении критических напряжений должны дефор- мироваться и частично разрушаться, чтобы при этом поглотить как можно большее количество кинетической энергии. Однако существует большое количество применений и для упругих градиентных сред. Например, их можно использовать для построения нелинейных механических осцилляторов со сложным поведением, поскольку здесь жесткость контакта всегда зависит от глубины индентирования даже в случае, когда площадь контакта остается постоянной величиной (например, при индентировании цилиндрического штемпеля).

Градиентные среды можно создавать послойно, напыляя покрытия с различными свойствами. Тогда свойства среды на границах раздела будут изменяться скачкообразно. Однако существуют такие материалы, свойства которых изменяются непрерывным образом. Примером этого служит костная ткань. Другой природный пример - адгезионная система лапы геккона, свойства которой за счет существующей иерархии также изменяются с глубиной $[7,8]$.

В недавней работе [5] было проведено изучение адгезии между жестким индентором, имеющим задающийся функцией произвольный профиль, и полупространством, которое представляет среду с градиентными свойствами. А именно рассмотрена ситуация, в которой модуль упругости $E$ является функцией глубины индентирова- 

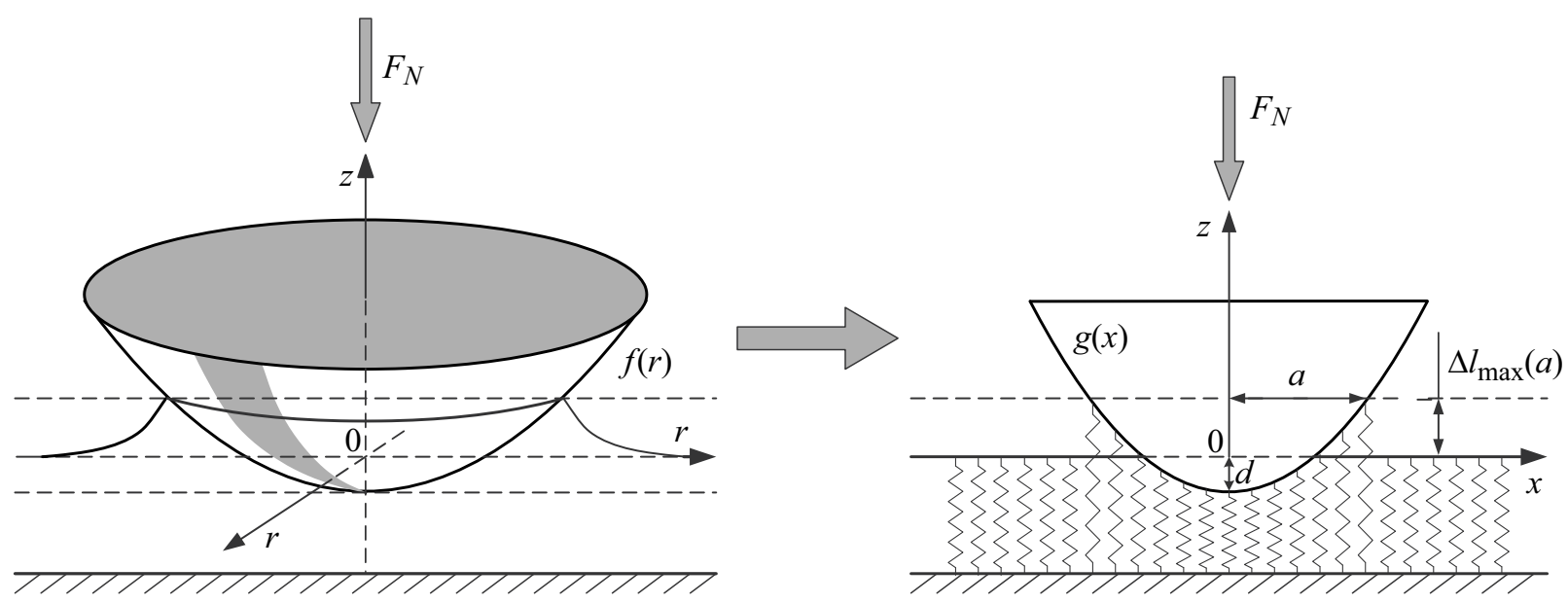

Рис. 1. Иллюстрация замены трехмерного профиля $f(r)$ эквивалентным упругим основанием, состоящим из невзаимодействующих пружин.

ния $z$. В этом случае зависимость модуля упругости от глубины индентирования имеет стандартный вид $[5,9,10]$ :

$$
E(z)=E_{0}\left(\frac{z}{c_{0}}\right)^{k}
$$

где $E_{0}-$ характерный модуль упругости, а $c_{0}-$ характерная длина.

В [5] рассматривается диапазон значений показателя степени $0<k<1$, поскольку полученное там решение определено только в этом интервале значений $k$. Однако для несжимаемых сред (коэффициент Пуассона $v=1 / 2$ ) получаемое решение при $k>1$ формально определено до $k<3$. Механические свойства градиентных сред со столь высоким показателем степени ранее уже рассматривались в работе [11]. Настоящая работа посвящена подробному изучению адгезионного контакта градиентных сред на примерах цилиндрического и параболического инденторов, в том числе с учетом шероховатостей в области контакта.

\section{Адгезионный контакт между цилиндром и полупространством}

Для описания адгезии между несжимаемым индентором и упругим градиентным полупространством будем использовать метод редукции размерности (method of dimensionality reduction - MDR), который позволяет точно описывать контактные задачи между аксиально симметричными телами с односвязным контактом $[12,13]$. Причем метод MDR успешно применяется для описания квазистатических и динамических контактов $[14,15]$. В недавних работах $[9,10]$ метод MDR был обобщен на случай градиентных сред, свойства которых задаются выписанным выше соотношением (1). В рамках метода сначала необходимо получить эквивалентный одномерный профиль индентора $g(x)$, который рассчитывается согласно обобщенному преобразованию Абеля $[9,14,16]$

$$
g(x)=|x|^{1-k} \int_{0}^{|x|} \frac{f^{\prime}(r)}{\sqrt{\left(x^{2}-r^{2}\right)^{1-k}}} \mathrm{~d} r,
$$

где $f(r)$ - радиальная функция, описывающая трехмерный профиль (рис. 1). Далее необходимо представить упругое полупространство в виде одномерного упругого основания, состоящего из независимых пружин с нормальной жесткостью $k_{z}=c_{W}(x) \Delta x(\Delta x-$ шаг дискретизации либо расстояние между пружинами). Функция $c_{W}(x)$ определяется выражением $[9,10]$

$$
c_{W}(x)=\frac{h(k, v)}{1-v^{2}} E(|x|)=\frac{h(k, v)}{1-v^{2}} E_{0}\left(\frac{|x|}{c_{0}}\right)^{k},
$$

где $v$ - коэффициент Пуассона, а функция $h(k, v)$ определяется группой соотношений $[5,10]$

$$
\begin{gathered}
h(k, v)=\frac{2(1+k) \cos \left(\frac{k \pi}{2}\right) \Gamma\left(1+\frac{k}{2}\right)}{\sqrt{\pi} C(k, v) \beta(k, v) \sin \left(\frac{\beta(k, v) \pi}{2}\right) \Gamma\left(\frac{1+k}{2}\right)}, \\
C(k, v)=\frac{2^{1+k} \Gamma\left(\frac{3+k+\beta(k, v)}{2}\right) \Gamma\left(\frac{3+k-\beta(k, v)}{2}\right)}{\pi \Gamma(2+k)}, \\
\beta(k, v)=\sqrt{(1+k)\left(1-\frac{k v}{1-v}\right)} .
\end{gathered}
$$

Коэффициент $h(k, v)$ для несжимаемых сред $(v=1 / 2), \quad$ которые будут рассматриваться в дальнейшем, может с достаточно необходимой точностью быть аппроксимирован соотношением [11]

$$
\begin{aligned}
h(k, v & =1 / 2) \approx 1.3666 \cdot \sin (0.9010 \cdot k+0.4997) \\
& +0.4727 \cdot \sin (1.8884 \cdot k+0.7664)
\end{aligned}
$$




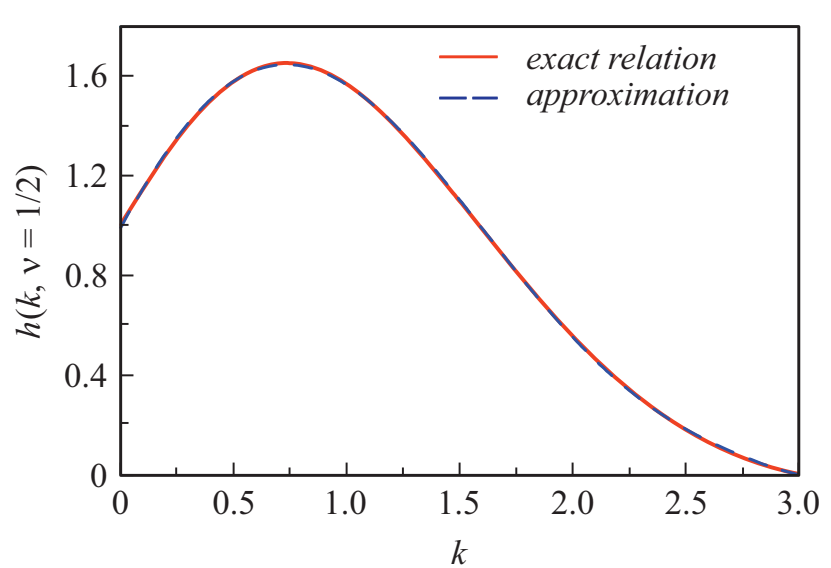

Рис. 2. Зависимость коэффициента $h(k, v)$ от показателя степени $k$ при заданном значении коэффициента Пуассона $v=1 / 2$.

Сравнение аппроксимации (5) с функцией $h(k, v)(4)$ при $v=1 / 2$ представлено на рис. 2.

Начнем с рассмотрения адгезионного контакта между цилиндрическим штемпелем с плоским основанием и градиентной средой, действуя в рамках описанного выше метода редукции размерности. При этом профиль цилиндра удобно представить в виде зависимости

$$
f(r)=r\left(\frac{r}{a_{0}}\right)^{N},
$$

где $r$ - радиальная координата, $a_{0}-$ радиус цилиндра, а показатель степени $N \rightarrow \infty$. Применение преобразования (2) к выражению (6) приводит к одномерной функции

$$
g(x)=\frac{(N+1) x^{N+1}}{2 a_{0}^{N} \Gamma\left(1+\frac{N+k}{2}\right)} \Gamma\left(\frac{1+k}{2}\right) \Gamma\left(\frac{N+1}{2}\right) .
$$

В работе [10] показано, что условие равновесия крайних пружин (рис. 1) в адгезионном контакте для градиентных сред дается формулой

$$
\Delta l_{\max }(a)=\sqrt{\frac{2 \pi \Delta \gamma a^{1-k} c_{0}^{k}}{E^{*} h(k, v)}},
$$

где $\Delta \gamma-$ поверхностная энергия (работа адгезии на единицу площади), $a$ - радиус контакта, а эффективный модуль упругости $E^{*}$ задается выражением $E^{*}=E_{0} /\left(1-v^{2}\right)$. Стоит отметить, что формула (8) справедлива для любой формы аксиально-симметричных тел, находящихся в односвязном контакте с полупространством. В случае цилиндра, для которого $g(x)$ задается функцией (7), глубина индентирования может быть определена из выражения

$$
\begin{aligned}
& d(a) \equiv g(a)-\Delta l_{\max }(a)=\frac{(N+1) a^{N+1}}{2 a_{0}^{N} \Gamma\left(1+\frac{N+k}{2}\right)} \\
& \times \Gamma\left(\frac{1+k}{2}\right) \Gamma\left(\frac{N+1}{2}\right)-\sqrt{\frac{2 \pi \Delta \gamma a^{1-k} c_{0}^{k}}{E^{*} h(k, v)}} .
\end{aligned}
$$

Действующая при этом нормальная сила $F_{N}(a)$ определяется как сумма сил от всех пружин, находящихся в контакте $[9,10]$ :

$$
F_{N}(a)=2 \int_{0}^{a} c_{W}(x)[d-g(x)] \mathrm{d} x,
$$

что немедленно приводит к результату

$$
\begin{aligned}
& F_{N}(a)=\frac{E^{*} h(k, v)(N+1)^{2}}{c_{0}^{k}(1+k)(2+k+N) \Gamma\left(1+\frac{N+k}{2}\right)} \Gamma\left(\frac{1+k}{2}\right) \\
& \times \Gamma\left(\frac{N+1}{2}\right) \frac{a^{2+k+N}}{a_{0}^{N}}-\sqrt{\frac{8 \pi a^{3+k} \Delta \gamma h(k, v) E^{*}}{(1+k)^{2} c_{0}^{k}}} .
\end{aligned}
$$

Критическое значение нормальной силы $F_{A}$, при которой происходит разрушение контакта (контакт становится неустойчивым), соответствует отрицательному значению глубины индентирования $d=-\Delta l_{\max }(a)$. Это выражение при использовании (10) приводит к результату [10]

$$
F_{A}=\sqrt{\frac{8 \pi a_{0}^{3+k} \Delta \gamma h(k, v) E^{*}}{(1+k)^{2} c_{0}^{k}}} .
$$

Для дальнейшего анализа с целью уменьшения количества параметров решение задачи (9), (11) удобно представить в безразмерных переменных. Как единицы измерения логично выбрать критические значения глубины индентирования $\Delta l_{\max }\left(a_{0}\right)$ (8) и нормальной силы $F_{A}(12)$. Решение адгезионной задачи (9), (11) при использовании безразмерных переменных

$$
\begin{gathered}
\tilde{F}_{N}=\frac{F_{N}}{F_{A}}, \quad \tilde{d}=\frac{d}{\Delta l_{\max }\left(a_{0}\right)}, \quad \tilde{a}=\frac{a}{a_{0}}, \\
\widetilde{\Delta \gamma}=\frac{2 \pi \Delta \gamma}{E^{*} h(k, v) a_{0}}, \quad \tilde{c}_{0}=\frac{c_{0}}{a_{0}}
\end{gathered}
$$

будет иметь вид

$$
\begin{aligned}
& \tilde{d}(\tilde{a})=\frac{(N+1)(\tilde{a})^{N+1}}{2 \Gamma\left(1+\frac{N+k}{2}\right) \sqrt{\tilde{c}_{0}^{k} \widetilde{\Delta \gamma}}} \Gamma\left(\frac{1+k}{2}\right) \Gamma\left(\frac{N+1}{2}\right) \\
& -\sqrt{(\tilde{a})^{1-k}}, \\
& \tilde{F}_{N}(\tilde{a})=\frac{(N+1)^{2}(\tilde{a})^{2+k+N}}{2(2+k+N) \Gamma\left(1+\frac{N+k}{2}\right) \sqrt{\tilde{c}_{0}^{k} \widetilde{\Delta \gamma}}} \\
& \times \Gamma\left(\frac{1+k}{2}\right) \Gamma\left(\frac{N+1}{2}\right)-\sqrt{(\tilde{a})^{3+k}} .
\end{aligned}
$$

Похожие выражения для произвольной аксиально симметричной геометрии контакта получены в работе [5], однако в указанной работе проводится подробное изучение случая параболического индентора и отсутствует описание особенностей адгезионных процессов для цилиндрического индентора. Анализируя выражения (14) 


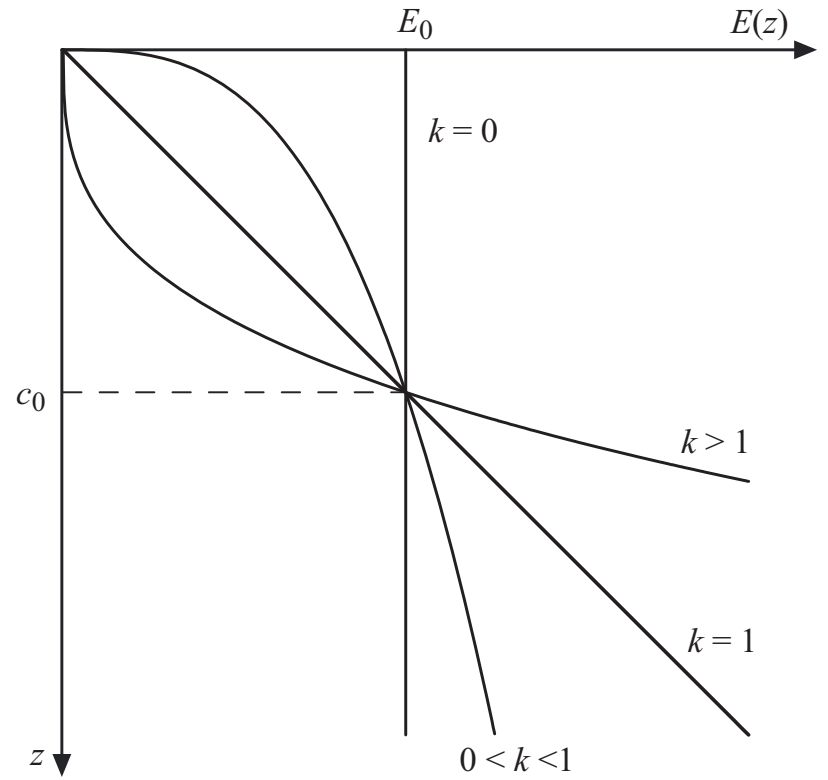

Рис. 3. Зависимости модуля упругости от глубины индентирования $E(z)(1)$ для различных значений показателя $k$.

и (15) при различных значениях показателя $k$ стоит помнить, что единицы измерения (13) также содержат параметр $k$. Поэтому на представленных далее графиках в безразмерных переменных сравнивая любые две зависимости при различных $k$, мы только можем сказать как изменяется нормальная сила по сравнению с ее критическим значением. Для того чтобы сравнивать кривые друг с другом при различных $k$, а именно делать вывод о том, приводит изменение параметра $k$ к увеличению или уменьшению нормальной силы либо прочности контакта, необходимо представить результаты в размерных значениях.

Величина параметра $k$ в выражении (1) выбирается из расчета того, что функция $h(k, v)(4)$ должна быть положительной. Эта функция для значений коэффициента Пуассона $0 \leq v<1 / 2$ положительна в области $0 \leq k<1$. При $k=1$ функция $h(k, v)$ принимает нулевое значение, что, согласно (3), соответствует нулевой жесткости среды, вне зависимости от глубины индентирования. При дальнейшем росте $k$ функция меняет знак, а жесткость при этом становится отрицательной, что не имеет физического смысла. Однако для несжимаемых сред при $v=1 / 2$ функция $h(k, v)$ положительна в более широкой области $0 \leq k<3$. В этом случае представляется возможным описать нетривиальное поведение, что является основной целью настоящей работы, поскольку случай $0 \leq k<1$ уже был подробно рассмотрен в работе [5] на примере параболического индентора. Стоит отметить, что при $v=1 / 2$ в области $k>1$ функция $\beta(k, 1 / 2)=\sqrt{1-k^{2}}$ становится мнимой величиной, однако $h(k, v)$ вне зависимости от типа значений $\beta(k, v)$ всегда действительна.
На рис. 3 показана зависимость модуля упругости $E(z)$ (1) для различных значений параметра $k$. Значение $k=0$ соответствует случаю постоянной жесткости $E(z)=E_{0}$. При описании градиентных сред ранее рассматривалась ситуация $0<k<1$ [5,9,10], когда в начале индентирования наблюдается бесконечное нарастание модуля упругости $\mathrm{d} E /\left.\mathrm{d} z\right|_{z \rightarrow 0} \rightarrow \infty$. В случае существенно более мягких материалов $(k>1)$ ситуация принципиально меняется, поскольку $\mathrm{d} E /\left.\mathrm{d} z\right|_{z \rightarrow 0} \rightarrow 0$. Выше мы указали, что для несжимаемой среды $v=1 / 2$ может быть рассмотрен диапазон значений $0 \leq k<3$, в котором функция $h(k, v)(4)$ сохраняет положительный знак, поэтому для исследования поведения системы в максимально широком диапазоне значений параметра $k$ в дальнейшем будем полагать $v=1 / 2$, хотя в безразмерных переменных (13) решение рассматриваемой контактной задачи $(14),(15)$ не зависит от значения функции $h(k, v)$. Однако при этом всегда стоит понимать, что случай $k>1$ реализуется только при $v=1 / 2$, поскольку в противоположном случае среда становится неустойчивой, а решение дает физически неправильные результаты, поскольку соответствует отрицательному значению контактной жесткости.

Зависимости нормальной силы $\tilde{F}_{N}$ и радиуса контакта $\tilde{a}$ от глубины индентирования $\tilde{d}$, рассчитанные по формулам (14) и (15), показаны на рис. 4. На этом рисунке легко видеть прямую, соответствующую зависимости силы от глубины индентирования в той области, в которой размер контакта не изменяется. Это, во-первых, положительные значения $\tilde{d}$, соответствующие вдавливанию, а также область отрицательных значений до начала потери устойчивости пружин на границе контакта. Из рисунка следует, что при $k<1$ (для $k<1$ справедливы ситуации с произвольным значением коэффициента Пуассона v) адгезионный контакт разрушается сразу, как только расстояние между полупространством и поверхностью цилиндра $|d|$ превышает критическое значение $\Delta l_{\max }\left(a_{0}\right)(8)\left(a_{0}-\right.$ радиус цилиндра). В этом случае при бо́льших значениях величины удаления равновесных состояний не существует.

В области вдавливания цилиндра и при удалении от полупространства вплоть до критической величины $|d|<\Delta l_{\text {max }}\left(a_{0}\right)$ согласно рисунку реализуется линейный участок зависимости $\tilde{F}_{N}(\tilde{d})$, поскольку в случае цилиндра при этом сохраняется постоянное значение площади контакта $a=a_{0}$. Из рис. 4 следует, что в случае $k>1$ (реализуемо только для $v=1 / 2$ ) после достижения критического расстояния $|d|>\Delta l_{\max }\left(a_{0}\right)$ и дальнейшем удалении цилиндра от полупространства радиус контакта уменьшается монотонно, асимптотически приближаясь к нулевому значению с удалением цилиндра. Это означает, что при контролируемом удалении цилиндра будет происходить постепенное уменьшение размера контакта и величины нормальной силы.

Отдельного внимания заслуживает предельный случай $k=1$, в котором функция $\beta(k, 1 / 2)=\sqrt{1-k^{2}}=0$. 

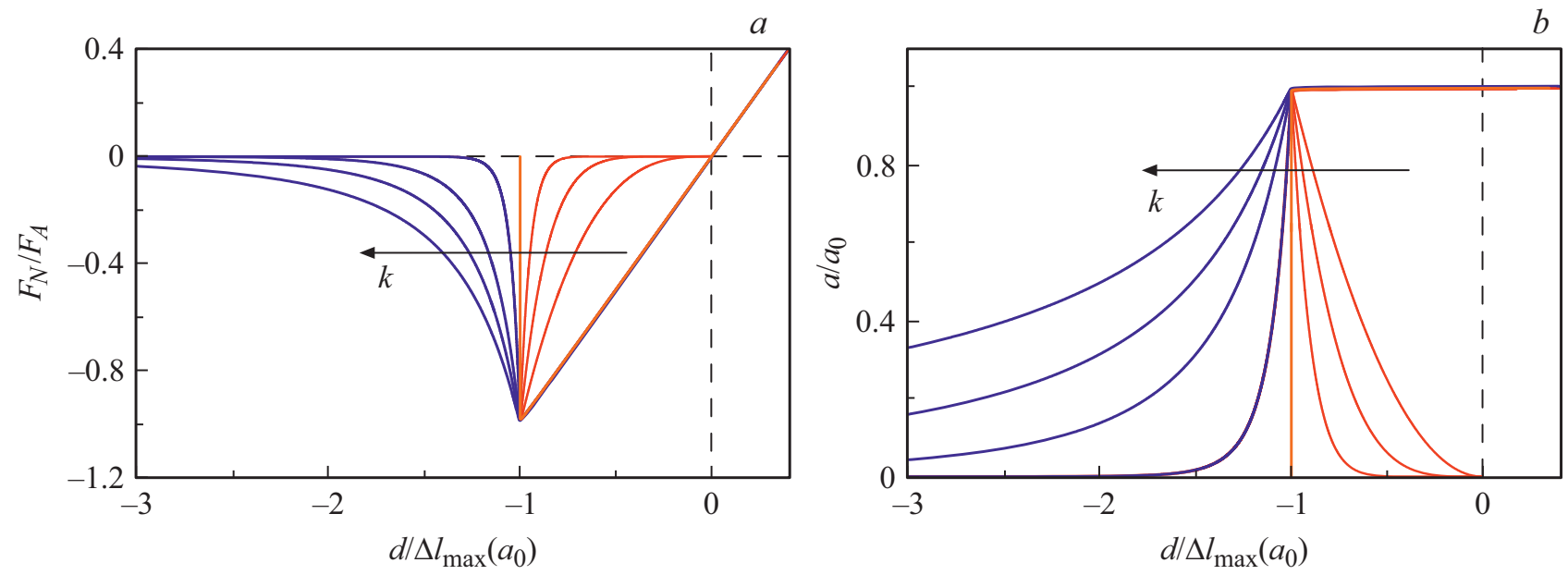

Рис. 4. $a$ - зависимости нормальной силы $\tilde{F}_{N}$ от глубины индентирования $\tilde{d}$, задающиеся соотношениями (14) и $(15)$ для значений показателя $k=0,0.5,0.8,1.0,1.2,1.7,2.2,2.99$ при параметрах $\widetilde{\Delta \gamma}=0.05, \tilde{c}_{0}=0.2$ и $N=1000 ; b-$ зависимости радиуса контакта $\tilde{a}$ от глубины индентирования $\tilde{d}$, соответствующие кривым, приведенным на рис. $4, a$. Направление увеличения показателя $k$ на обоих рисунках показано стрелкой.
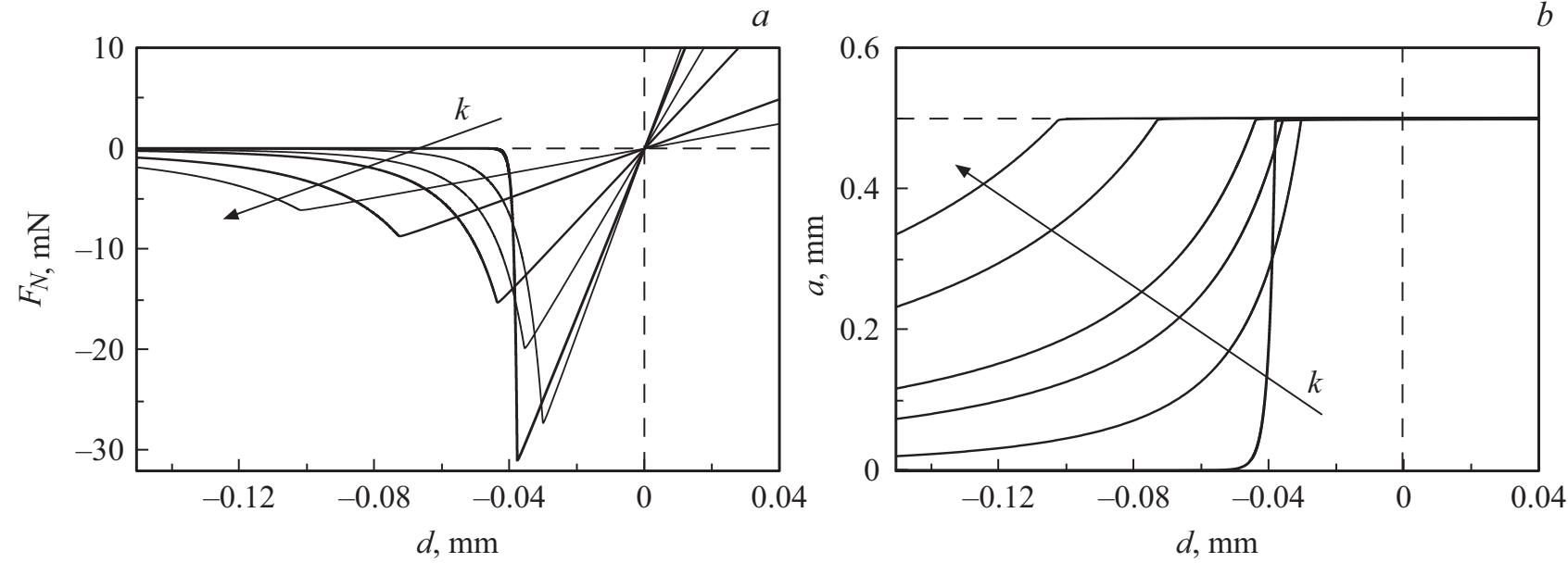

Рис. 5. $a$ - зависимости нормальной силы $F_{N}$ от глубины индентирования $d$ при параметрах $a_{0}=0.5 \cdot 10^{-3} \mathrm{~m}, E^{*}=0.2 \cdot 10^{6} \mathrm{~Pa}$, $c_{0}=10^{-4} \mathrm{~m}, \Delta \gamma=0.7958 \mathrm{~J} / \mathrm{m}^{2} ; b$ - зависимости радиуса контакта $a$ от глубины индентирования $d$, соответствующие кривым на рис. 5, $a$. Кривые на обоих рисунках соответствуют параметрам $k=1.1,2.0,2.5,2.7,2.9,2.95$, увеличение параметра $k$ показано стрелкой.

При этом в определении функции $h(k, 1 / 2)$ (4) появляется неопределенность $\cos (k \pi / 2) / \sqrt{1-k^{2}}$, которая, однако, в точке $k=1$ дает значение 1 , а значение функции $h(1,1 / 2)=\pi / 2$, поэтому факт неопределенности не вызывает противоречий. Из рис. 4 видно, что при $k=1$ реализуется ситуация, в которой отрыв цилиндра также происходит в случае $|d|>\Delta l_{\max }\left(a_{0}\right)$.

Таким образом, значение $k=1$ разграничивает физически качественно различное поведение: если $k<1$, контакт при выполнении условия $|d|>\Delta l_{\max }\left(a_{0}\right)$ разрушается мгновенно, а в случае $k>1$ при превышении удалением $|d|$ максимального значения $|d|>\Delta l_{\max }\left(a_{0}\right)$ происходит монотонное уменьшение радиуса контакта $a$ с дальнейшим ростом $|d|$ (рис. $4, b$ ).

Из рис. 4 можно было бы сделать вывод о том, что с увеличением значения $k$ для отрыва цилиндра нужно прикладывать бо́льшие силы, однако это утверждение является неочевидным, поскольку, как мы указали выше, единицы измерения содержат показатель $k$. В этом случае для сравнивания величин прикладываемой силы необходимо анализировать зависимости $F_{N}(d)$ в размерных значениях. Для проведения такого сравнения приведем аналогичные зависимости, но уже в размерном виде. В качестве параметров выберем радиус цилиндра $a_{0}=0.5 \cdot 10^{-3} \mathrm{~m}$ и модуль упругости $E^{*}=0.2 \cdot 10^{6} \mathrm{~Pa}$. Поскольку мы хотим построить зависимости, которые полностью соответствовали бы параметрам рис. 4, недостающие размерные параметры $\Delta \gamma$ и $c_{0}$ необходимо определить из соотношений (13), которые устанавливают связь между размерными и безразмерными параметрами. Используя уже выбранные нами размерные величины и параметры, при которых построен рис. 4, 

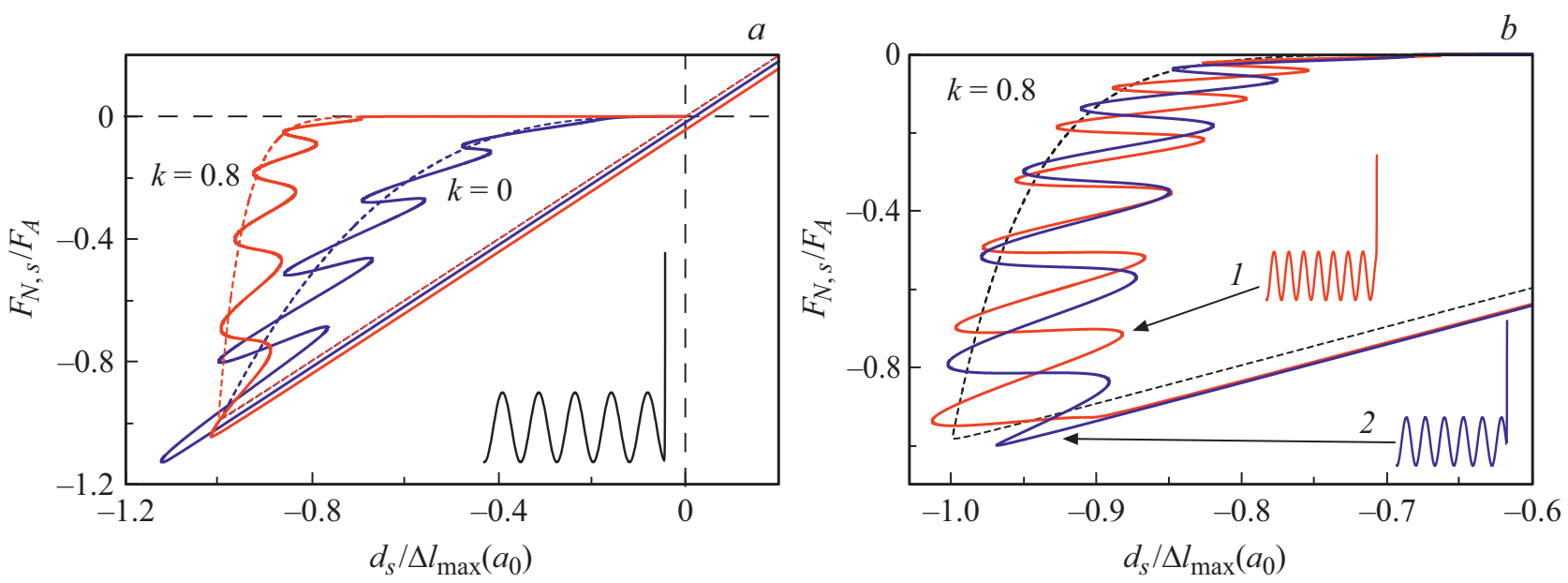

Рис. 6. Зависимости нормальной силы $\tilde{F}_{N, s}$ от глубины индентирования $\tilde{d}_{s}$, задающиеся соотношениями (17) и (18) при параметрах рис. 4 и $\tilde{h}=0.005: a-\tilde{\lambda}=0.2, k=0$ и $k=0.8 ; b-k=0.8(\tilde{\lambda}=0.134$ для кривой 1 и $\tilde{\lambda}=0.172$ для кривой 2$)$. Штриховыми линями для сравнения показано решение задачи при тех же параметрах, но при $\tilde{h}=0$ (плоский цилиндр).

будем иметь $c_{0}=10^{-4} \mathrm{~m}$ и $\Delta \gamma \approx 0.7958 \mathrm{~J} / \mathrm{m}^{2}$. Полученные при этих параметрах зависимости для $k>1$ показаны на рис. 5, который демонстрирует более сложное поведение, чем зависимости в безразмерных параметрах на рис. 4. Интересным здесь является то, что с увеличением показателя $k$ разрушение контакта начинается при меньших силах, однако эти меньшие критические значения силы соответствуют бо́льшим значениям глубины индентирования $|d|$. Затем с увеличением удаления индентора от полупространства $|d|$ ситуация изменяется - бо́льшим $k$ соответствуют бо́льшие значения нормальной силы $\left|F_{N}\right|$, т. е. в рассматриваемом случае при выбранных параметрах система показывает сложную нелинейную зависимость критических величин от параметра $k$. Однако при этом не стоит забывать, что мы построили рис. 5 при фиксированных параметрах модели и при их изменении картина может существенно отличаться. Для выяснения всех особенностей поведения системы при изменении параметра $k$ необходимо проводить полный многопараметрический анализ, который не является целью настоящей работы. Однако при необходимости такой анализ может быть проведен используя записанные выше аналитические соотношения.

Особый интерес представляет случай, в котором поверхности контактирующих тел имеют шероховатости [17-19]. В рамках метода MDR может быть рассмотрен только контакт аксиально симметричных тел, поэтому в роли шероховатости целесообразно выбрать радиально симметричную функцию. В $[5,20]$ проведено такое исследование на примере параболоида, шероховатость которого задавалась функцией

$$
f_{s}(r)=h\left[1-\cos \left(\frac{2 \pi r}{\lambda}\right)\right]
$$

значения которой прибавлялись к изначальному профилю $f(r)$, определенному функцией типа (6). В выражении (16) $h$ - амплитуда, $\lambda$ - длина волны, $r-$ радиальная координата. Исследуем здесь влияние таким образом определенной „шероховатости“ для цилиндра. Если к изначальному профилю $f(r)$ (6) прибавить функцию $f_{s}(r)(16)$ и произвести все описанные выше вычисления, то решение задачи адгезии цилиндра с полупространством принимает вид

$$
\begin{aligned}
& \tilde{d}_{s}(\tilde{a})=\tilde{d}(\tilde{a})+\frac{(\tilde{a})^{1-\frac{k}{2}} \cdot \pi^{\frac{3-k}{2}} \cdot \tilde{h}}{(\tilde{\lambda})^{1-\frac{k}{2}} \sqrt{\tilde{c}_{0}^{k} \widetilde{\Delta \gamma}}} \cdot H\left(\frac{k}{2}, \frac{2 \pi \tilde{a}}{\tilde{\lambda}}\right) \Gamma\left(\frac{1+k}{2}\right), \\
& \tilde{F}_{N, s}(\tilde{a})=\tilde{F}_{N}(\tilde{a})+\frac{(1+k) \cdot \pi^{\frac{1-k}{2}} \cdot \tilde{h}}{2 \sqrt{\tilde{c}_{0}^{k} \widetilde{\Delta \gamma}}} \\
& \times \Gamma\left(\frac{1+k}{2}\right)(\tilde{\lambda})^{\frac{k}{2}}(\tilde{a})^{1+\frac{k}{2}} \\
& \times\left[\frac{2 \pi \tilde{a}}{(k+1) \tilde{\lambda}} \cdot H\left(\frac{k}{2}, \frac{2 \pi \tilde{a}}{\tilde{\lambda}}\right)-H\left(1+\frac{k}{2}, \frac{2 \pi \tilde{a}}{\tilde{\lambda}}\right)\right] . \\
& \text { В (17), (18) введена функция Струве } H(n, x)[13,21]
\end{aligned}
$$

$$
H(n, x)=\sum_{m=0}^{\infty} \frac{(-1)^{m}}{\Gamma\left(m+\frac{3}{2}\right) \Gamma\left(m+n+\frac{3}{2}\right)}\left(\frac{x}{2}\right)^{2 m+n+1}
$$

и появляются две дополнительные безразмерные переменные $\tilde{\lambda}=\lambda / a_{0}$ и $\tilde{h}=h / a_{0}$. Функции $\tilde{d}(\tilde{a})$ и $\tilde{F}_{N}(\tilde{a})$, как и ранее, определяются соотношениями (14) и (15). Решение (17), (18) при фиксированных параметрах показано на рис. 6 . Из рис. $6, a$ следует, что в случае $k<1$, несмотря на волнистую поверхность цилиндра, так же как и для плоского цилиндра (рис. 4), разрушение контакта происходит, когда расстояние $\left|d_{s}\right|$ превышает критическое значение. Однако при наличии волнистости, как следует из рисунка, для отрыва цилиндра от поверхности необходимо приложить большее значение нормальной силы $F_{N, s}$. Стоит отметить, что с ростом 


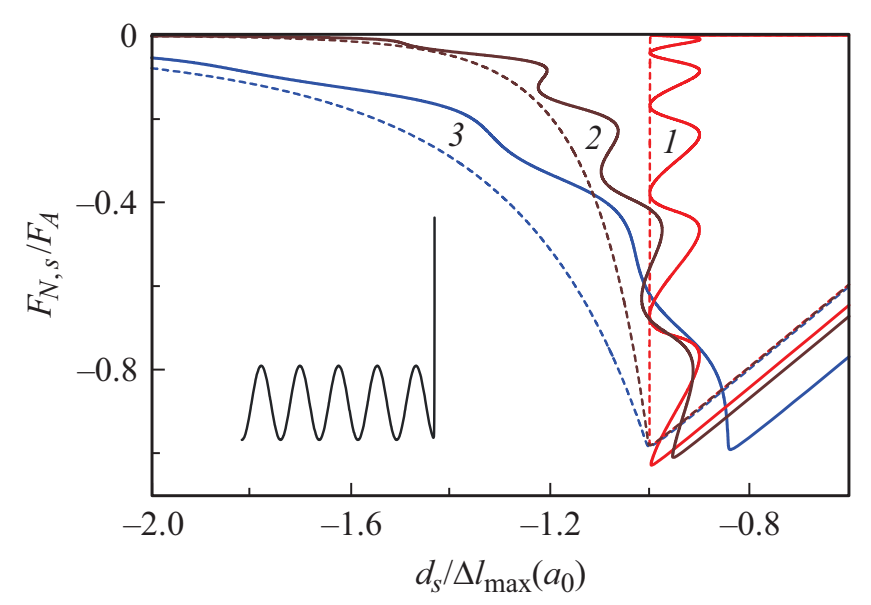

Рис. 7. Зависимости нормальной силы $\tilde{F}_{N, s}$ от глубины индентирования $\tilde{d}_{s}$, задающиеся соотношениями (17) и (18) при параметрах рис. $6, a$; группы кривых 1-3 соответствуют значениям $k=1,1.5,2.5$. Штрихом показаны аналогичные зависимости при отсутствии волнистости $(\tilde{h}=0)$.

значения показателя $k$ влияние волнистости (шероховатости) уменьшается, что видно из сравнения кривых на рис. $6, a$. Причем при наличии волнистости нулевой глубине индентирования $\tilde{d}=0$ соответствует ненулевое значение нормальной силы $\tilde{F}_{N, s}$.

На рис. 6, a показана ситуация, когда контакт становится неустойчивым при достижении величиной $\left|d_{s}\right|$ некоторого максимального значения, после чего происходит отрыв цилиндра. Однако в зависимости от соотношения длины волны $\lambda$ и радиуса цилиндра $a_{0}$ (у нас это отношение задается величиной безразмерного параметра $\left.\tilde{\lambda}=\lambda / a_{0}\right)$ на краях цилиндра будут реализоваться геометрические особенности, которые качественно влияют на адгезионное поведение при удалении цилиндра от полупространства. Для выяснения этих особенностей на рис. 6 дополнительно показаны половинные сечения профилей цилиндра, которые представляют сумму функций $f(r)+f_{s}(r)$ в диапазоне $r \in\left[0, a_{0}\right)$. Так, на рис. $6, b$ выделено две ситуации. Рассмотрим сначала ситуацию, отвечающую профилю под номером 1 на рисунке. Здесь, согласно кривой 1, уменьшение радиуса контакта начинается раньше, чем в случае гладкого цилиндра (зависимость $\tilde{F}_{N, s}\left(\tilde{d}_{s}\right)$ раньше отклоняется от линейного участка зависимости, что соответствует уменьшению радиуса контакта). Далее при превышении величиной $\left|d_{s}\right|$ критической величины происходит полное разрушение контакта. В другом выделенном случае (профиль под номером 2) при увеличении расстояния между цилиндром и полупространством существует две критические точки - первый раз контактный радиус резко уменьшается, что приводит к снижению абсолютного значения нормальной силы $\tilde{F}_{N, s}$, но после этого появляется другая устойчивая конфигурация контакта, для разрушения которой необходимо снова отдалять цилиндр от полупространства. При превышении величиной $\left|d_{s}\right|$ второго критического значения контакт исчезает полностью. В работе [5], в которой изучался контакт параболического индентора и полупространства, таких устойчивых конфигураций контакта может быть несколько, и их количество увеличивается с уменьшением длины волны $\lambda$. В случае же цилиндра, как нами показано выше, возможны только две ситуации - либо контакт исчезает сразу при превышении расстоянием $\left|d_{s}\right|$ критического значения, либо реализуются два критических значения $\left|d_{s}\right|$.

Более интересная ситуация возникает при значении параметра $k>1$, соответствующие зависимости $\tilde{F}_{N, s}\left(\tilde{d}_{s}\right)$ показаны на рис. 7. Кривые 1 на этом рисунке построены при значении показателя $k=1$, при котором при достижении расстоянием $\left|d_{s}\right|$ критического значения реализуется ситуация „безразличного равновесия“, поскольку все экстремумы функции $\tilde{F}_{N, s}\left(\tilde{d}_{s}\right)$, соответствующие устойчивым конфигурациям контакта, находятся на единственной прямой $\tilde{d}_{s}=-1$. При увеличении показателя $k$ (кривые 2) реализуется ситуация, когда радиус контакта при увеличении $\left|d_{s}\right|$ уменьшается скачкообразно, поскольку существует несколько устойчивых конфигураций, что для случая параболического индентора справедливо также и в области $k<1$, как это показано в работе [5]. Однако если показатель $k$ превышает некоторое критическое значение (группа кривых 3), радиус контакта при достижении величиной $\left|d_{s}\right|$ критического значения уменьшается монотонно. В последнем случае конфигурация контакта для всех стационарных значений $\tilde{d}_{s}$ становится устойчивой.

\section{Адгезионный контакт между параболоидом и полупространством}

Хотя случай параболического индентора был подробно рассмотрен в работе [5], в ней проведено исследование для диапазона значений $k<1$. Выясним здесь, какие особенности в поведение адгезионного контакта вносит ситуация $k>1$, справедливая при $v=1 / 2$. Параболический индентор с наличием аксиально-симметричных волнистых „шероховатостей“ описывается функцией

$$
f_{p}(r)=\frac{r^{2}}{2 R}+h\left[1-\cos \left(\frac{2 \pi r}{\lambda}\right)\right],
$$

где $R$ - радиус индентора. После введения безразмерных параметров

$$
\begin{gathered}
\tilde{F}_{N, p}=\frac{F_{N, p}}{F_{0}}, \quad \tilde{d}_{p}=\frac{d_{p}}{d_{0}}, \quad \tilde{a}=\frac{a}{a_{0}}, \\
\tilde{\lambda}=\frac{\lambda}{a_{0}}, \quad \tilde{h}=\frac{h}{d_{0}}, \quad \tilde{c}_{0}=\frac{c_{0}}{a_{0}},
\end{gathered}
$$

где $[3,13]$

$$
\begin{gathered}
F_{0}=\frac{3}{2} \pi R \Delta \gamma, \quad a_{0}=\left(\frac{9 \pi R^{2} \Delta \gamma}{8 E^{*}}\right)^{1 / 3}, \\
d_{0}=\left(\frac{3 \pi^{2} R \Delta \gamma^{2}}{64 E^{* 2}}\right)^{1 / 3},
\end{gathered}
$$

Журнал технической физики, 2020, том 90, вып. 5 

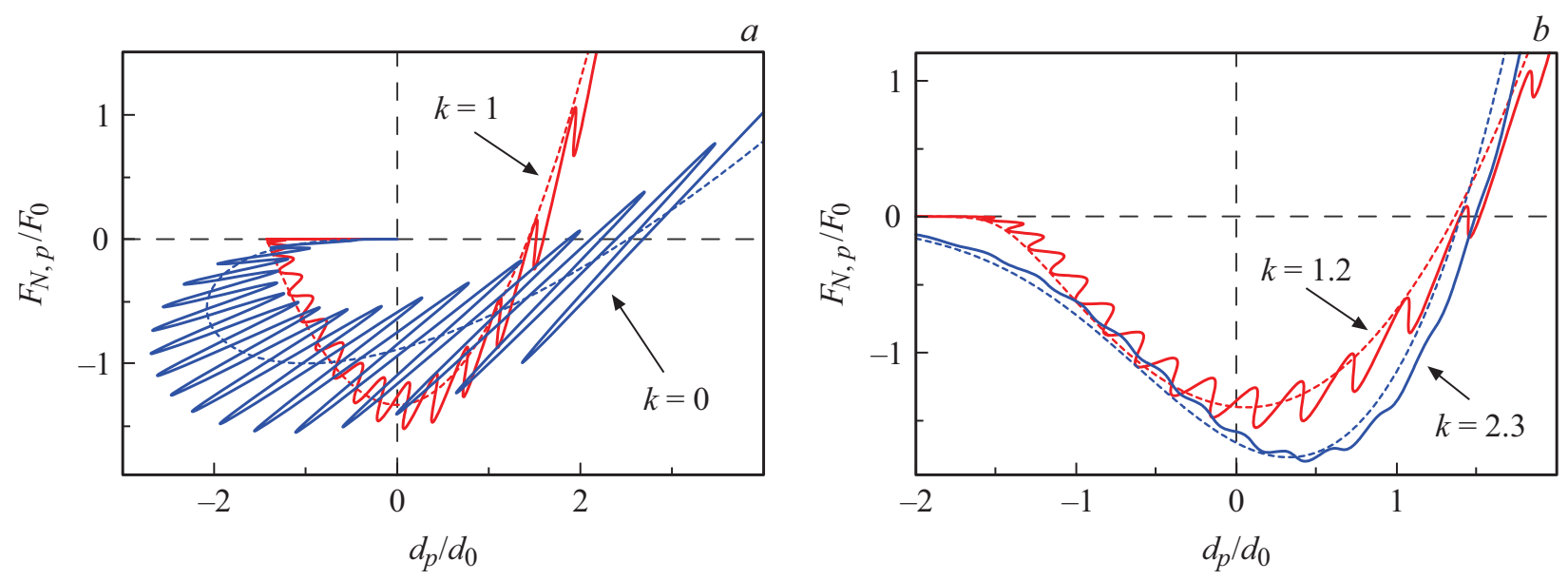

Рис. 8. Зависимости нормальной силы $\tilde{F}_{N, p}$ от глубины индентирования $\tilde{d}_{p}$, задающиеся соотношениями (20) и (21) при параметрах $v=1 / 2, \tilde{h}=0.1, \tilde{\lambda}=0.1, \tilde{c}_{0}=0.2: a-k=0$ и $k=1 ; b-k=1.2$ и $k=2.3$. Штриховыми линиями показаны те же зависимости, только при отсутствии шероховатостей $(\tilde{h}=0)$.

использование функции (19) приводит к решению

$$
\begin{aligned}
& \tilde{d}_{p}(\tilde{a})=\frac{3(\tilde{a})^{2}}{1+k}-4 \sqrt{\frac{(\tilde{a})^{1-k}\left(\tilde{c}_{0}\right)^{k}}{h(k, v)}} \\
& +\pi^{\frac{3-k}{2}}\left(\frac{\tilde{a}}{\tilde{\lambda}}\right)^{1-\frac{k}{2}} \tilde{h} \Gamma\left(\frac{1+k}{2}\right) H\left(\frac{k}{2}, \frac{2 \pi \tilde{a}}{\tilde{\lambda}}\right), \\
& \tilde{F}_{N, p}(\tilde{a})=\frac{3 h(k, v)(\tilde{a})^{3+k}}{\tilde{c}_{0}^{k}(1+k)^{2}(3+k)}-2 \sqrt{\frac{(\tilde{a})^{3+k} h(k, v)}{(1+k)^{2} \tilde{c}_{0}^{k}}} \\
& +\frac{h(k, v)}{4 \tilde{c}_{0}^{k}} \Gamma\left(\frac{1+k}{2}\right) \pi^{\frac{1-k}{2}}(\tilde{\lambda})^{\frac{k}{2}}(\tilde{a})^{1+\frac{k}{2}} \tilde{h} \\
& \times\left[\frac{2 \pi \tilde{a}}{(k+1) \tilde{\lambda}} H\left(\frac{k}{2}, \frac{2 \pi \tilde{a}}{\tilde{\lambda}}\right)-H\left(1+\frac{k}{2}, \frac{2 \pi \tilde{a}}{\tilde{\lambda}}\right)\right] .
\end{aligned}
$$

Заметим, что в решении для цилиндра (17), (18) нормальная сила $\tilde{F}_{N}$ и глубина индентирования $\tilde{d}$ измерены в максимальных величинах $F_{A}(12)$ и $\Delta l_{\max }\left(a_{0}\right)(8)$, соответствующих возникновению неустойчивости контакта для плоского цилиндра при произвольном значении показателя $k$. Этот факт на показанных выше зависимостях $\tilde{F}_{N}(\tilde{d})$ приводит к тому, что отрыв плоского цилиндра $(\tilde{h}=0)$, либо уменьшение радиуса контакта всегда начинается в точке $(-1,-1)$. Для параболоида мы нормировали величины на стандартные значения, используемые в теории JKR [3]. Такие единицы измерения имеют определенные преимущества - поскольку они не содержат параметр $k$, можно однозначно сравнивать поведение системы при его различных значениях, основываясь непосредственно на безразмерных зависимостях $\tilde{F}_{N}(\tilde{d})$.

На рис. 8, а показано решение задачи для двух значений $k=0$ и $k=1$. Мы не будем останавливаться на описании особенностей такого поведения, поскольку оно было подробно описано в работе [5]. Однако стоит отметить, что в предельном случае $k=1$ в отсутствие шероховатостей $(\tilde{h}=0)$ решение $\tilde{F}_{N, p}\left(\tilde{d}_{p}\right)$ становится устойчивым на всем диапазоне глубин индентирования $\tilde{d}_{p}$. Это приводит к тому, что радиус контакта при увеличении расстояния $\left|d_{p}\right|$ между параболическим индентором и полупространством уменьшается постепенно, вплоть до нулевого значения. Подобная ситуация наблюдается и в случае $k>1$ (рис. 8,b), с тем отличием, что при $k>1$ с увеличением $\tilde{d}_{p}$ нормальная сила и радиус контакта к нулевым значениям с ростом $\left|d_{p}\right|$ приближаются асимптотически. Как это видно из рис. 8 , с ростом показателя $k$ влияние шероховатости уменьшается, что подтверждает сделанные ранее выводы для цилиндрического индентора. При необходимости исследование, проведенное в настоящей работе, можно обобщить на случай произвольной аксиально симметричной геометрии контакта с произвольной радиально симметричной функцией, задающей шероховатость. Для этого достаточно выбрать соответствующие выражения для $f(r)$ и $f_{s}(r)$, после чего произвести описанные выше вычисления.

\section{Заключение}

В работе проведено аналитическое решение контактной задачи цилиндрического и параболического индентора с полупространством, представляющим градиентную среду, для которой модуль упругости увеличивается с глубиной индентирования. Решение получено в безразмерных переменных, поэтому все кривые (зависимости нормальной силы от глубины индентирования) являются универсальными. При этом дополнительно проведен учет волнистости („шероховатости“) контактной поверхности, которая задается радиальной функцией. Показано, что с ростом показателя степени, задающего градиентность, влияние неровностей поверхностей на свойства адгезионного контакта уменьшается. Изучена 
ситуация, в которой с ростом удаления индентора от полупространства контакт монотонно и асимптотически приближается к нулевому значению. Причем это реализуемо как для параболического, так и для цилиндрического индентора. Такое поведение возможно только для несжимаемых сред (коэффициент Пуассона 1/2), для которых показатель степени $k$, задающий градиентные свойства среды, может принимать значения больше единицы.

Таким образом, высокий показатель степени $k$ градиентности среды может привести к качественному изменению адгезионного поведения как гладких, так и шероховатых контактов. Так, для гладких цилиндрических контактов значение $k=1$ разграничивает случаи мгновенного, неустойчивого разрушения контакта от постепенного, непрерывного. Соответственно, при высоких степенях градиентности резко повышается работа разрушения адгезионного контакта.

\section{Благодарности}

Авторы благодарны Ли Чиангу (Li Qiang) и Маркусу Xeccy (Markus Heß) за плодотворные дискуссии.

\section{Финансирование работы}

Работа выполнена при финансовой поддержке Немецкого научно-исследовательского сообщества (DFG), проект PO 810-55-1; частично содержит результаты исследований, проведенных в рамках гранта президента Украины по конкурсному проекту 0119U103175.

\section{Конфликт интересов}

Авторы заявляют, что у них нет конфликта интересов.

\section{Список литературы}

[1] Pocius A.V. Adhesion and adhesives technology: An introduction (Third Edition). Munich, Germany: Carl Hanser Verlag, 2012. $370 \mathrm{p}$.

[2] Johnson K.L, Kendall K., Roberts A.D. // Proc. Royal Soc. Lond. A. 1971. Vol. 324. N 1558. P. 301-313.

[3] Johnson K.L. // Proc. R. Soc. Lond. A. 1997. Vol. 453. N 1956. P. $163-179$.

[4] Maugis D. Contact, adhesion, and rupture of elastic solids. Berlin, Germany: Springer Berlin Heidelberg, 2000. 414 p.

[5] Jin F., Guo X. // Int. J. Solids Struct. 2013. Vol. 50. N 20-21. P. 3375-3386.

[6] Willert E., Lyashenko I.A., Popov V.L. // Comput. Part. Mech. 2018. Vol. 5. N 3. P. 313-318.

[7] Scherge M., Gorb S. Biological micro- and nanotribology. Nature's solutions. N. Y.: Springer-Verlag, 2001. 304 p.

[8] Chen S., Yan C., Soh A. // Int. J. Solid Struct. 2009. Vol. 46. N 18-19. P. 3398-3404.

[9] Heß M. // Int. J. Eng. Sci. 2016. Vol. 104. P. 20-33.
[10] Hess M., Popov V.L. // Facta Univ., Ser.: Mech. Eng. 2016. Vol. 14. N 3. P. 251-268.

[11] Popov V.L. // Phys. Mesomech. 2018. Vol. 21. N 1. P. 76-79.

[12] Popov V.L., Hess M. // Facta Univ., Ser.: Mech. Eng. 2014. Vol. 12. N 1. P. 1-14.

[13] Popov V.L., Heß M. Method of dimensionality reduction in contact mechanics and friction. Berlin, Germany: Springer Verlag, 2015. 265 p.

[14] Willert E., Dmitriev A.I., Psakhie S.G., Popov V.L. // Sci. Rep. 2019. Vol. 9. P. 7791.

[15] Lyashenko I.A., Borysiuk V.N., Popov V.L. // Meccanica. 2019. Vol. 54. N 10. P. 1681-1687.

[16] Argatov I. // Facta Univ., Ser.: Mech. Eng. 2019. Vol. 17. N 2. P. 181-190.

[17] Persson B.N.J. // Surf. Sci. Rep. 2006. Vol. 61. N 4. P. 201-227.

[18] Ciavarella M., Papangelo A. // J. Adhes. Sci. Technol. 2017. Vol. 31. N 22. P. 2445-2467.

[19] Martina D., Creton C., Damman P., Jeusette M., Lindner A. // Soft Matter. 2012. Vol. 8. N 19. P. 5350-5357.

[20] Jacobs T.D.B., Ryan K.E., Keating P.L., Grierson D.S., Lefever J.A., Turner K.T., Harrison J.A., Carpick R.W. // Trib. Lett. 2013. Vol. 50. N 1. P. 81-93.

[21] Struve H. // Annalen der Physik. 1882. Vol. 253. N 13. P. 1008-1016. 